\title{
A Simple Method of Determining Pre-Diabetes
}

\author{
Joon Hyuck Choi ${ }^{1}$ and Nam Lyong $\mathrm{Kang}^{*}{ }^{2}$
}

${ }^{1}$ Department of Internal Medicine, Hankuk Hospital, Osan 447-804, Republic of Korea (Previous Address: Department
of Internal Medicine, Catholic University of Daegu, Daegu 712-702, Republic of Korea)

${ }^{2}$ Department of Nano Medical Engineering, Pusan National University, Miryang 627-706, Republic of Korea

\begin{abstract}
A simple formula for determining pre-diabetes (the very early stage of diabetes mellitus) was derived from the model of Ackerman et al. [Bull Math Biophys 1965; 27: s21-37] for very smoothly varying time course patterns of blood glucose level. The natural period of blood glucose level in a healthy subject calculated using the formula was in good agreement with the value obtained by computer work.
\end{abstract}

Keywords: Pre-diabetes, blood glucose level, natural period.

Diabetes mellitus (DM) is a disease associated with disordered metabolism of carbohydrates, and pre-diabetes mellitus (PD) is a very early stage of mild DM caused by disordered secretion of insulin, glucagons and epinephrine. The study of PD is of great importance in opening a way to the early discovery of mild DM. Although quite many approaches to DM and PD on mathematical basis have been reported thus far [1-6], most of them require advanced mathematical and computer work ability. The purpose of this report was to provide a simple method of determining PD in order to help general practitioners with limited mathematical and computer ability. This study was based on a simple model presented by Ackerman et al. [1,7], which was based on the simple assumption that the blood glucose level tends to be regulatory and is influenced and controlled by a wide variety of hormones and other metabolites.

In this model all these hormones are combined into a net hormonal concentration and the cumulative effect of them is taken into account. And thus the model centers attention on the concentration of glucose in the blood, labelled $G$ and that of the net hormonal complex, labelled $H$. The hormones such as insulin which decrease $G$ are considered to increase $H$. On the other hand, the materials such as glucagons, epinephrine and cortisol which increase $G$ are considered to decrease $H$. Coupled linear differential equations for $G$ and $H$ can be established and these two first order equations can be combined into two separate forms of second order linear equation. The solution for $G$, with $t=0$ at the time the glucose loading has been completed, is generally given in three damped oscillation patterns. Among them, in analogy with mechanical vibration systems, the underdamped case seems to be more appropriate for $\mathrm{PD}$, by assuming that there is no serious deficiency in the controlling system. Thus we have (Fig. 1)

$G(t)=G_{0}+A \exp \left[-\lambda\left(t-t_{\mathrm{m}}\right)\right] \cos \left[\omega\left(t-t_{\mathrm{m}}\right)\right]$

*Address correspondence to this author at the Department of Nano Medical Engineering, Pusan National University, miryang 627-706, Republic of Korea; E-mail: nlkang@pusan.ac.kr for $t \geq 0$, where we have rearranged the phase factor in such a way that the maximum value $G_{\mathrm{m}}=G_{0}+A\left(G_{0}>A>0\right)$ is given around the time $t=t_{\mathrm{m}}$. Note that the original equation used by Ackerman et al. [1,7] can be replaced by Eq. (1) for very smoothly varying time course patterns of $G(t)$. We can have a similar form for $H$, which is not shown here. It should be noted that there are four constants $\left(A, \lambda, \omega, t_{\mathrm{m}}\right)$ for the solutions. For very smoothly varying function $G(t)$, this modified form turns out to be fairly well accepted (The original equation is given as Eq. (4) in Table 1).

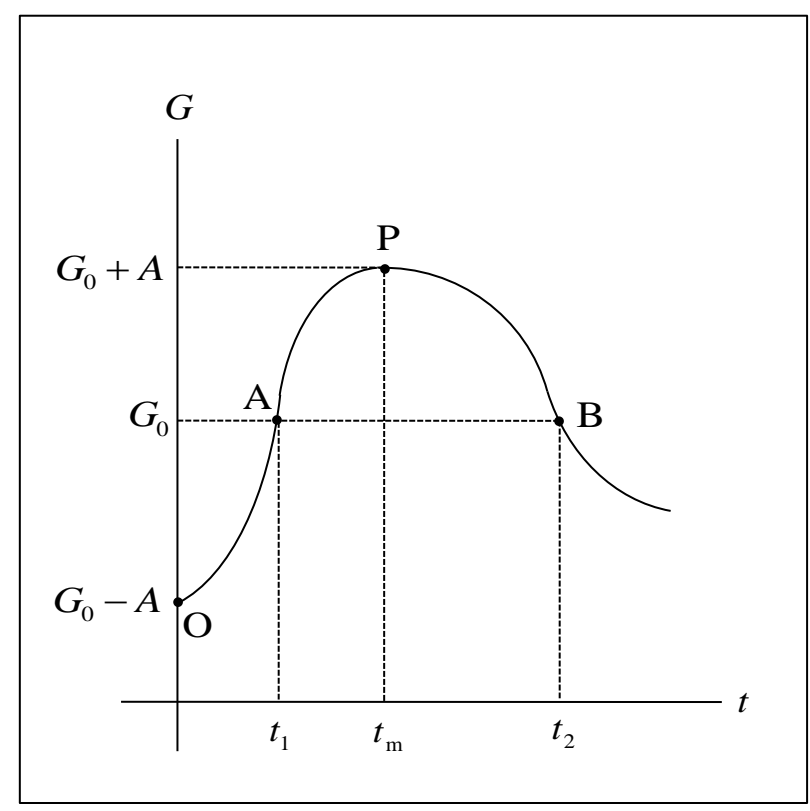

Fig. (1). $G-t$ profile of PD.

$t=0$ : the time at which moderate amount of $G(t)$ is given.

$t=t_{\mathrm{m}}$ : the time for the maximum point $\mathrm{P}$.

$t=t_{1}$ and $t=t_{2}:$ the times for point $\mathrm{A}$ and point $\mathrm{B}$, respectively, on the abscissa $\mathrm{AB}$ [the half way between the minimum point $\mathrm{O}$ and the maximum point $\mathrm{P}]$. 
Table 1. Blood Glucose Levels for a Healthy Subject (M/65, Korean, Weight: 57 kg). After Overnight Fasting, Moderate Amount of Glucose was Given at $t=0$

\begin{tabular}{|llllllllllll}
$t[\mathrm{~h}]$ & 0 & 0.2 & 0.5 & 0.8 & 1.0 & 1.2 & 1.5 & 1.7 & 2.0 & 2.5 & 3.1 \\
$G[\mathrm{mg} / \mathrm{dL}]$ & 79 & 101 & 120 & 139 & 145 & 145 & 130 & 125 & 110 & 95 & 90
\end{tabular}

(a) the model of Ackerman et al: : $G=G_{0}+B \exp (-\lambda t) \cos (\omega t-\delta)$

From this regression equation, we have

$G_{0}=105 \mathrm{mg} / \mathrm{dL}, B=75 \mathrm{mg} / \mathrm{dL}, \lambda=0.58 / \mathrm{h}, \omega=1.74 / \mathrm{h}, \delta=2 \mathrm{rad}$

which gives the natural period $T_{0}=2 \pi\left(\lambda^{2}+\omega^{2}\right)^{-1 / 2}=3.4 \mathrm{~h}$

(b) the present method :

$$
\begin{aligned}
& t_{\mathrm{m}}=1.1 \mathrm{~h}, t_{1}=0.3 \mathrm{~h}, t_{2}=2.1 \mathrm{~h} \text { from Fig. (1) } \\
& \theta=\pi t_{\mathrm{m}} /\left(t_{2}-t_{1}\right)=1.92 \mathrm{rad}=110 \text { degrees from Eq. (3) } \\
& T_{0}=2 \pi t_{\mathrm{m}}\left\{\theta^{2}+[\ln (-\cos \theta)]^{2}\right\}^{-1 / 2}=3.1 \mathrm{~h} \text { from Eq. (2) }
\end{aligned}
$$

In the model of Ackerman et al. the phase parameters $\lambda$ and $\omega$ are measured by fitting the equation [Eq. (4)] to the data available. The natural period $T_{0}$ defined as $T_{0}=2 \pi / \omega_{0}$ where $\omega_{0}=\left(\lambda^{2}+\omega^{2}\right)^{1 / 2}$ is the key factor. The model declares that a value of less than 4 hours for $T_{0}$ indicates normalcy, while appreciably more than 4 hours implies PD. Note that the usual meal period is about 4 hours $[1,7]$. The merit of this model is that only the data for $G$ are needed in determining PD.

Now we will derive a simple formula which can be utilized without help of computer work. We let $G\left(t_{1}\right)=G\left(t_{2}\right)=G_{0}$ for time $t_{1}$ and $t_{2}\left(t_{2}>t_{1}\right)$. Then from Eq. (1) we have $\omega\left(t_{1}-t_{\mathrm{m}}\right)=-\pi / 2$ and $\omega\left(t_{2}-t_{\mathrm{m}}\right)=\pi / 2$ which yield $\omega=\pi /\left(t_{2}-t_{1}\right)$. On the other hand, as $G(0)=G_{0}-A$, we have $\exp \left(\lambda t_{\mathrm{m}}\right) \cos \omega t_{\mathrm{m}}=-1$ which yields $\lambda=\left(-1 / t_{m}\right) \ln \left(-\cos \omega t_{m}\right)$ where $\ln x \equiv \log _{\mathrm{e}} x$ is the natural logarithm of $x$. Note that both $\omega$ and $\lambda$ are positive constants. The natural angular frequency $\omega_{0}$ can be determined in terms of the three characteristic times $t_{1}, t_{2}$ and $t_{\mathrm{m}}$ which can be measured from the $G$ - $t$ profile. In this simplified model, the optimum value $G_{0}$ can be determined graphically by the abscissa $A B$ which is half way between the minimum point $\mathrm{O}$ and the maximum point $\mathrm{P}$ (Fig. 1).

The final formula for the natural period of PD with very smoothly varying time course profile of $G(t)$ is now given by

$T_{0}=2 \pi t_{\mathrm{m}}\left\{\theta^{2}+[\ln (-\cos \theta)]^{2}\right\}^{-1 / 2}$

where the characteristic angle $\theta$ is given by

$\pi / 2<\theta=\pi t_{\mathrm{m}} /\left(t_{2}-t_{1}\right)<3 \pi / 2$.

So far we have introduced a simple method of measuring the natural period $T_{0}$ from the elaborately drawn $G-t$ profile. In view of the characteristics in relation with the ability of regulation, $\lambda^{2}$ and $\omega_{0}^{2}$, respectively, may be called "autoregulatance" and "cross-regulatance". The underdamped oscillation is specified by the condition $\omega^{2}=\omega_{0}^{2}-\lambda^{2}$ [7]. In most cases we have experienced for subjects, however, $\omega^{2}>\lambda^{2}$. If $\omega^{2} \gg \lambda^{2}, T_{0} \cong 2\left(t_{2}-t_{1}\right)$ which is identical to the period of simple harmonic oscillation.

Now this method is applied to data taken on a healthy subject as shown in Table $\mathbf{1}$ for the sake of demonstration. According to Eqs. (2)-(3) and the figure drawn using the data given in Table 1 (the figure is not shown here), $t_{\mathrm{m}}=1.1 \mathrm{~h}$, $t_{1}=0.3 \mathrm{~h}$ and $t_{2}=2.1 \mathrm{~h}$, and thus the condition [Eq. (3)] for this approximation holds $(\pi<2 \theta<3 \pi)$. From Eq. (2) we have $T_{0}=3.1 \mathrm{~h}$. On the other hand, by performing computer work based on Eq. (4) we have $\omega=1.74 / \mathrm{h}$ and $\lambda=0.58 / \mathrm{h}$ yielding $T_{0}=3.4 \mathrm{~h}$. The two results are in good agreement with each other. As $T_{0}<4$ hours, this subject is free from PD according to the criterion of Ackerman et al.

This method may be applicable to very early discovery of type $2 \mathrm{DM}$ in subjects with family history for the following reason. DM is partly inherited and the inheritance pattern is stronger for type 2 . The patients with first-degree relatives with type 2 have a much higher risk of developing type 2 [8]. The classical triad of diabetes symptoms (polyurea, polydipsia, and polyphagia) usually develop more rapidly in children with type 1 diabetes. On the other hand, in most patients with type 2 PD the symptoms are absent, and thus the early determination is almost impossible. Therefore, if the pedigree systems are well managed, it will be not so difficult to select the subjects for whom the early diagnosis of DM is needed. This simple method, which is a simplified version of the model of Ackerman et al. for very smoothly varying time course patterns of $G(t)$, can be used by any practitioners understanding elementary arithmetic, since only the time course data for blood glucose levels are required. Thus in conclusion we claim that the present technique will be used as a subsidiary tool for determination of PD.

\section{REFERENCES}

[1] Ackerman E, Gatewood LC, Rosevear JW, Molnar GD. Model studies of blood-glucose regulation. Bull Math Biophys 1965; 27: s21-37.

[2] Bergman RN, Ider YZ, Bowden CR, Cobelli C. Quantitative estimation of insulin sensitivity. Am J Physiol 1979; 236: E667-77. 
[3] Celeste R, Ackerman E, Gatewood LC, Reynolds C, Molnar GD. The role of glucose in the regulation of blood glucose: model studies. Bull Math Biol 1978; 40: 59-77.

[4] Jansson L, Lindskog L, Norden NE. Diagnostic value of the oral glucose tolerance test evaluated with a mathematical model. Comp Biomed Res 1980; 13: 512-21.

[5] De Gaetano A, Arino O. Mathematical modeling of the intravenous glucose tolerance test. J Math Biol 2000; 40: 136-68.
[6] Zheng Y, Zhao M. Modified minimal model using a single-step fitting process for the intravenous glucose tolerance test in Type 2 diabetes and healthy humans. Comput Meth Prog Biomed 2005; 79: 73-9.

[7] Braun M. Differential Equations and Their Applications. $4^{\text {th }}$ ed. New York: Springer 1993; Chapter 2.

[8] Rother KI. Diabetes treatment-bridging the divide. N Engl J Med 2007; 356: 1499-501.

(C) Choi and Kang; Licensee Bentham Open.

This is an open access article licensed under the terms of the Creative Commons Attribution Non-Commercial License (http://creativecommons.org/licenses/by$\mathrm{nc} / 3.0 /$ ) which permits unrestricted, non-commercial use, distribution and reproduction in any medium, provided the work is properly cited. 